\title{
Characterization of Fusobacterium nucleatum ATCC 23726 adhesins involved in strain-specific attachment to Porphyromonas gingivalis
}

\author{
Jane Park, Bhumika Shokeen, Susan K Haake and Renate Lux
}

Bacterial adherence is an essential virulence factor in pathogenesis and infection. Fusobacterium nucleatum has a central role in oral biofilm architecture by acting as a bridge between early Gram-positive and late Gram-negative colonizers that do not otherwise adhere to each other. In this study, we survey a key adherence interaction of $F$. nucleatum with Porphyromonas gingivalis, and present evidence that multiple fusobacterial adhesins have a role in the attachment of $F$. nucleatum ATCC 23726 to $P$. gingivalis in a highly strain-dependent manner. Interaction between these species displayed varying sensitivities to arginine, galactose and lactose. Arginine was found to hamper coaggregation by at least $62 \%$ and up to $89 \%$ with several $P$. gingivalis strains and galactose inhibition ranged from no inhibition up to $58 \%$ with the same $P$. gingivalis strains. Lactose consistently inhibited $F$. nucleatum interaction with these $P$. gingivalis strains ranging from $40 \%$ to $56 \%$ decrease in coaggregation. Among the adhesins involved are the previously described Fap2 and surprisingly, RadD, which was described in an earlier study for its function in attachment of $F$. nucleatum to Gram-positive species. We also provide evidence for the presence of at least one additional adhesin that is sensitive to arginine but unlike Fap2 and RadD, is not a member of the autotransporter family type of fusobacterial large outer membrane proteins. The strain-specific binding profile of multiple fusobacterial adhesins to $P$. gingivalis highlights the heterogeneity and complexity of interspecies interactions in the oral cavity.

International Journal of Oral Science (2016) 8, 138-144; doi:10.1038/ijos.2016.27; published online 19 August 2016

Keywords: adhesin; biofilm; coaggregation; Fusobacterium nucleatum; Porphyromonas gingivalis

\section{INTRODUCTION}

Fusobacterium nucleatum is a prevalent member of the oral microbial community and considered a key organism in biofilm formation due to its ability to adhere to a large variety of microbial species. ${ }^{1-3}$ Although present in healthy oral biofilms, this Gram-negative opportunistic pathogen is dominant in periodontal disease $\mathrm{e}^{4}$ and has been implicated in a number of invasive human infections, ${ }^{5-6}$ acute and chronic inflammatory conditions ${ }^{7-8}$ as well as adverse pregnancy outcomes ${ }^{9-10} \mathrm{~F}$. nucleatum pathogenicity is, in part, attributed to its function as a "bridging organism" that supports the integration of periodontal pathogens into oral biofilms. ${ }^{2,11}$ With this unique ability to attach to both early and late colonizers, $F$. nucleatum is thought to have a central role in the ecological shift from a mostly Gram-positive to a predominately Gram-negative and thus, pathogenic, biofilm community in periodontal disease. ${ }^{2}$

Despite the extensive exploration of $F$. nucleatum interspecies interactions and the identification of a number of binding partners, to date, only two fusobacterial large outer membrane proteins (OMPs), RadD and Fap2, have been characterized at a molecular level for their role as adhesins in binding to a variety of Gram-positive species ${ }^{12-13}$ and Porphyromonas gingivalis, respectively. ${ }^{14}$ Both RadD and Fap2 are members of the autotransporter family of proteins, ${ }^{13}$ which are the largest known family of virulence factors expressed by Gram-negative bacteria. ${ }^{15}$ Autotransporters account for numerous biological functions including adhesion ${ }^{16-17}$ cell-to-cell aggregation, ${ }^{18-19}$ biofilm formation ${ }^{20-21}$ and invasion. ${ }^{22}$ It is therefore not surprising that in addition to their role in interspecies binding, fusobacterial autotransporters are multifunctionally involved in the induction of apoptosis in lymphocytes ${ }^{23-24}$ and adherence to murine placental cells. ${ }^{14}$

Historically, studies have shown that interbacterial binding measured by the classical coaggregation assay often involves highly serotype or strain-specific cell-to-cell recognition. ${ }^{25}$ For example, a panel of different F. nucleatum strains and several species of Selenomonas were found to bind only to certain subsets of oral partner species tested but no distinct group-specific pattern was observed. ${ }^{2}$ In particular, adhesion of different $F$. nucleatum isolates to a selection of $P$. gingivalis strains varied from no interaction to very strong coaggregation phenotypes, some of which were sensitive to lactose or heat treatment, while others were not. ${ }^{26}$ This interspecies binding variation is not limited to fusobacterial interactions but appears to be a common theme among oral bacterial species. Previous studies of different oral bacterial interactions have demonstrated that coaggregation involves highly

School of Dentistry, University of California at Los Angeles, Los Angeles, USA

Correspondence: Dr R Lux, School of Dentistry, University of California at Los Angeles, 10833 Le Conte Avenue, Los Angeles CA 90095, USA

E-mail: rlux@dentistry.ucla.edu

Accepted 25 March 2016 
specific cell-to-cell recognition of distinct isolates of a certain species and that this pattern is not generalizable to all strains of a single species or all species of a genus. Other examples of these differential binding specificities include Actinomyces naeslundii with different strains of Streptococcus sanguinis and Streptococcus gordonii, ${ }^{25,27-29}$ P. gingivalis binding with Veillonella, Capnocytophaga and Actinomyces but not with Streptococcus, ${ }^{30}$ intra- and intergenic coaggregations between Streptococcus and Actinomyces ${ }^{31}$ among others.

Interactions between $F$. nucleatum and $P$. gingivalis are of key interest because they are frequently isolated together from several chronic immunoinflammatory diseases of the oral cavity. ${ }^{32-33}$ While identification of Fap2 as the galactose-inhibitable adhesin of F. nucleatum strains ATCC 23726 for binding to P. gingivalis strain PK 1924 provided the first molecular identification of a fusobacterial adhesin involved in this interaction, ${ }^{14}$ previous reports of differential binding between various strains of these species ${ }^{26}$ as well as the finding that their coaggregation can be inhibited by carbohydrates other than galactose indicated the presence of additional adhesins. In this study, we tested the coaggregation between F. nucleatum strain ATCC 23726 and five strains of $P$. gingivalis, characterized the carbohydrate sensitivity of the interaction and screened the existing panel of autotransporter mutants in ATCC 23726 (ref. 13) for possible binding defects. This led to the identification of RadD as an additional strainspecific fusobacterial adhesin for interaction with $P$. gingivalis as well as the finding that further adhesins exist that do not belong to the autotransporter family of proteins despite being inhibited by the addition of arginine similar to RadD.

\section{MATERIALS AND METHODS}

\section{Bacterial strains and culture conditions}

F. nucleatum strain ATCC 23726 and its mutant derivatives defective in large outer membrane autotransporter proteins ${ }^{13,24}$ as well as seven different $P$. gingivalis strains 4612 (ref. 34), T22 (ref. 35), MP4-504 (ref. 36), ATCC 33277 (ref. 35), 381 (ref. 37), W50 and W83 (ref. 38), were maintained on Columbia agar supplemented with $5 \%$ sheep blood or in Columbia broth (CB; Difco, Detroit, MI, USA) under anaerobic conditions $\left(10 \% \mathrm{H}_{2}, 10 \% \mathrm{CO}_{2}, 80 \% \mathrm{~N}_{2}\right)$ at $37^{\circ} \mathrm{C}$. All media for $P$. gingivalis were also supplemented with hemin at $5 \mu \mathrm{g} \cdot \mathrm{mL}^{-1}$ and menadione at $1 \mu \mathrm{g} \cdot \mathrm{mL}^{-1}$. Thiamphenicol at $5 \mu \mathrm{g} \cdot \mathrm{mL}^{-1}$ and clindamycin at $1 \mu \mathrm{g} \cdot \mathrm{mL}^{-1}$ (MP Biomedicals, Irvine, CA, USA) were used for the selection and maintenance of strains possessing the catP and ermB determinants, respectively.

\section{Coaggregation assay}

Visual. Coaggregation assays were performed in coaggregation buffer $\left(\mathrm{CAB} ; 150 \mathrm{mmol} \cdot \mathrm{L}^{-1} \mathrm{NaCl}, 1 \mathrm{mmol} \cdot \mathrm{L}^{-1}\right.$ Tris, $0.1 \mathrm{mmol} \cdot \mathrm{L}^{-1} \mathrm{CaCl}_{2}$, $\left.0.1 \mathrm{mmol} \cdot \mathrm{L}^{-1} \mathrm{MgCl}_{2} \cdot \mathrm{H}_{2} \mathrm{O} ; \mathrm{pH} 7.5\right)$ as previously described. ${ }^{13}$ In brief, cells were pelleted and re-suspended in $\mathrm{CAB}$ to a final concentration of $2 \times 10^{9}$ cells per $\mathrm{mL}$ (optical density (OD) measured at $600 \mathrm{~nm}$ was 2 ). Suspensions of strains to be examined for coaggregation were combined with an equal volume of a test strain adjusted to the same cellular concentration in $\mathrm{CAB}$ to a total volume of $400 \mu \mathrm{L}$ in a reaction tube. Once the second partner strain was added, reaction mixtures were immediately vortexed for $5 \mathrm{~s}$ and incubated for at least $10 \mathrm{~min}$ prior to evaluation using a visual scoring system ranging from 0 to 4 . $^{3}$ A score of 0 was assigned for no visible coaggregation and a score of 4 described complete sedimentation of strains with a clear supernatant (Supplementary Table S1).

Quantitative. Coaggregation assays were performed identical to the visual assay except that ODs of reaction mixtures were obtained spectrophotometrically immediately after the addition of second partner strain and vortexing $\left(\mathrm{OD}_{t=0 \mathrm{~min}}\right)$. After $10 \mathrm{~min}$ of incubation, reaction mixtures were centrifuged at low speed ( $100 \mathrm{~g}$ for $1 \mathrm{~min}$ ) to pellet coaggregated cells while leaving non-aggregated bacteria in suspension. ODs of the supernatants were measured after the 10-min incubation $\left(\mathrm{OD}_{t=10 \mathrm{~min}}\right)$ in order to quantitate coaggregation. Coaggregation test reactions were calculated as $\left(\mathrm{OD}_{t=0 \min }-\mathrm{OD}_{\mathrm{t}=10 \mathrm{~min}}\right) /$ $\mathrm{OD}_{t=0 \text { min. }}{ }^{39}$ These values were averaged across at least three independent experiments and represented as percentages calculated relative to control reactions (reactions without the addition of a partner strain) as $\left(\right.$ Avg $\left._{\text {test reaction }} / \mathrm{Avg}_{\text {control reaction }}\right) \times 100$.

Inhibition. For both visual and quantitative inhibition assays, either L-arginine, D-galactose, L-glutamic acid, D-glucose or lactose was added to the reaction tube containing only $F$. nucleatum cells to a final concentration of $100 \mathrm{mmol} \cdot \mathrm{L}^{-1}$. The suspension was then vortexed and incubated for $5 \mathrm{~min}$ prior to the addition of the coaggregation test partner. Once the partner strain was added, the reaction mixture was vortexed again and the assay was evaluated via the quantitative coaggregation assay as described above. The final concentration of each inhibitor per coaggregation reaction was $50 \mathrm{mmol} \cdot \mathrm{L}^{-1}$.

\section{Biofilm growth}

Dual species biofilms. The F. nucleatum and P. gingivalis biofilm growth protocol was modified from a previous study ${ }^{40}$ by using 96-well collagen-coated plates (Advanced BioMatrix, San Diego, CA, USA) that were ultraviolet sterilized for $1 \mathrm{~h}$ prior to inoculation. For dual-species biofilms, $100 \mu \mathrm{L}$ of $\mathrm{CB}$ supplemented with hemin at $5 \mu \mathrm{g} \cdot \mathrm{mL}^{-1}$ and menadione at $1 \mu \mathrm{g} \cdot \mathrm{mL}^{-1}$ were added into each well. F. nucleatum cells $\left(50 \mu \mathrm{L}\right.$ containing $\sim 5 \times 10^{7}$ cells $)$ were seeded into each well and allowed to grow under anaerobic conditions $\left(10 \% \mathrm{H}_{2}\right.$, $10 \% \mathrm{CO}_{2}, 80 \% \mathrm{~N}_{2}$ ) at $37^{\circ} \mathrm{C}$ for $3 \mathrm{~h}$ prior to the addition of $P$. gingivalis $\left(50 \mu \mathrm{L}\right.$ containing $\sim 5 \times 10^{7}$ cells) to allow $F$. nucleatum cells time to bind to and saturate the collagen-coated surfaces. Plates were incubated overnight under anaerobic conditions $\left(10 \% \mathrm{H}_{2}, 10 \%\right.$ $\mathrm{CO}_{2}, 80 \% \mathrm{~N}_{2}$ ) at $37^{\circ} \mathrm{C}$. Triplicate wells were inoculated for each experiment, which were combined for DNA extraction. At least three biological replicates were performed per condition.

Fusobacterial attachment. To ensure that each of the fusobacterial strains attached to collagen-coated wells over $3 \mathrm{~h}$, after growth under anaerobic conditions $\left(10 \% \mathrm{H}_{2}, 10 \% \mathrm{CO}_{2}, 80 \% \mathrm{~N}_{2}\right)$ at $37^{\circ} \mathrm{C}$, contents were removed from each well and rinsed once with $250 \mu \mathrm{L}$ of sterile phosphate-buffered saline. Plates were inverted and dried. Afterwards, attached bacteria were fixed at room temperature for $15 \mathrm{~min}$ by adding $200 \mu \mathrm{L}$ of methanol into each well. The plates were stained with a $100 \mu \mathrm{L}$ aqueous solution of $0.5 \%$ crystal violet (Thermo Fisher Scientific, Waltham, MA, USA) for $15 \mathrm{~min}$ at room temperature. The plates were then carefully rinsed with Millipore water (Darmstadt, Germany) until there was no visible trace of the stain. Bound stain was dissolved by adding $160 \mu \mathrm{L}$ of $95 \%$ ethanol. The OD of each well was measured at $570 \mathrm{~nm}$ and was represented as relative to a negative control wells that only contained CB (Supplementary Figure S1). At least three biological replicates were performed per F. nucleatum strain.

\section{Extraction of DNA from biofilms}

Prior to DNA isolation, the medium was carefully removed from the wells. Genomic DNA was isolated directly from the wells using GenElute Bacterial Genomic DNA Kit (Sigma-Aldrich, St Louis, MO, USA) according to manufacture's instructions with the modi- 
fication of final elution to $30 \mu \mathrm{L}$. Buffers, enzymes and precipitating DNA were directly added into the wells prior to combining replicate samples and transferring the solution to the columns.

\section{Quantitative (real-time) polymerase chain reaction}

To quantify the relative proportions of each species in the respective dual-species biofilms, species-specific primer pairs were used. For F. nucleatum ATCC 23726 and its mutant derivatives, a portion of the Fusobacterium-specific fomA gene was amplified with Fn-F (forward) 5'-AGTTGCTCCAGCTTGGAGACCAAAT-3' and Fn-R (reverse) 5'-AAGTTTACTTTTGTTAAAGTTTGTAATCTTCC-3' primers. For P. gingivalis Pg-F (forward) 5'-AGGCAGCTTGCCATACTGCG-3' and Pg-R (reverse) 5'-ACTGTTAGCAACTACCGATGT-3' were chosen to amplify a portion of the $P$. gingivalis $16 \mathrm{~S} r R N A$ gene. Primer pairs were tested for possible cross-reactivity with the other species. Real-time quantitative polymerase chain reaction ( $\mathrm{qPCR}$ ) was performed using an iCycler Thermal Cycler (Bio-Rad, Hercules, CA, USA) in a total volume of $20 \mu \mathrm{L}$ containing $10 \mu \mathrm{L}$ of $2 \times$ iQ SYBR Green Supermix (Bio-Rad, Hercules, CA, USA), $0.5 \mu \mathrm{mol} \cdot \mathrm{L}^{-1}$ each of forward and reverse primers, $7 \mu \mathrm{L}$ of Millipore water and $1 \mu \mathrm{L}(10 \mathrm{ng})$ of template DNA. Amplification and detection were carried out in 96-well optical plates (Thermo Fisher Scientific, Waltham, MA, USA). Each PCR run was carried out with an initial incubation of $10 \mathrm{~min}$ at $95^{\circ} \mathrm{C}$ followed by 40 cycles of denaturing at $95^{\circ} \mathrm{C}$ for $15 \mathrm{~s}$; annealing and elongation at $60^{\circ} \mathrm{C}$ for $1 \mathrm{~min}$. After the 40 cycles of amplification, an additional denaturing step was performed at $95^{\circ} \mathrm{C}$ for $1 \mathrm{~min}$ followed by annealing and elongation at $60^{\circ} \mathrm{C}$ for $1 \mathrm{~min}$. A melting curve analysis was completed after each run. The DNA concentrations $\left(\mathrm{ng} \cdot \mathrm{mL}^{-1}\right.$ ) were calculated with standard curves obtained by 10 -fold serial dilutions of bacterial genomic DNA. Three independent qPCR runs were performed with three technical replicates for each sample to assess reproducibility and inter-run variability.

\section{Statistical analysis}

Student's $t$-test was performed to determine statistical significance using Excel 2011 (Microsoft, Seattle, WA, USA).

\section{RESULTS}

Autoaggregation of bacteria and coaggregation of $F$. nucleatum ATCC 23726 with $P$. gingivalis displays a strain-specific profile Qualitative and quantitative autoaggregation and coaggregation assays revealed a strain-dependent binding profile of $F$. nucleatum ATCC 23726 with the seven different strains of $P$. gingivalis tested in this study. Robust interactions were observed with $P$. gingivalis strains 4612 ( $62 \% \pm 8 \%$ coaggregation), T22 (54\% $\pm 8 \%$ coaggregation) binding and ATCC $33277(65 \% \pm 17 \%$ coaggregation; Figure 1). Binding of $P$. gingivalis strains W50 and W83 to F. nucleatum ATCC 23726 was notably weaker, showing only $23 \% \pm 10 \%$ and $30 \% \pm 22 \%$ coaggregation, respectively. $P$. gingivalis strains 381 and the clinical isolate MP-504 revealed $54 \% \pm 2 \%$ and $73 \% \pm 4 \%$ coaggregation, respectively, but were not chosen for further interaction studies with F. nucleatum due to notable autoaggregation levels. Interactions of fusobacteria with other species can be disrupted by a number of small molecules including arginine, galactose and lactose. ${ }^{26}$ Attachment to early colonizers was described to be largely inhibitable by arginine ${ }^{41}$ with the identification of RadD as the arginine-inhibitable adhesin for streptococci, ${ }^{13}$ whereas adhesion between F. nucleatum and Gramnegative late colonizers including $P$. gingivalis was found to be generally sensitive to galactose. ${ }^{26,42}$ Fap2 was recently identified as the galactose-inhibitable adhesin for this interaction. ${ }^{14}$ Interestingly, the binding profile of $F$. nucleatum strain ATCC 23726 with the

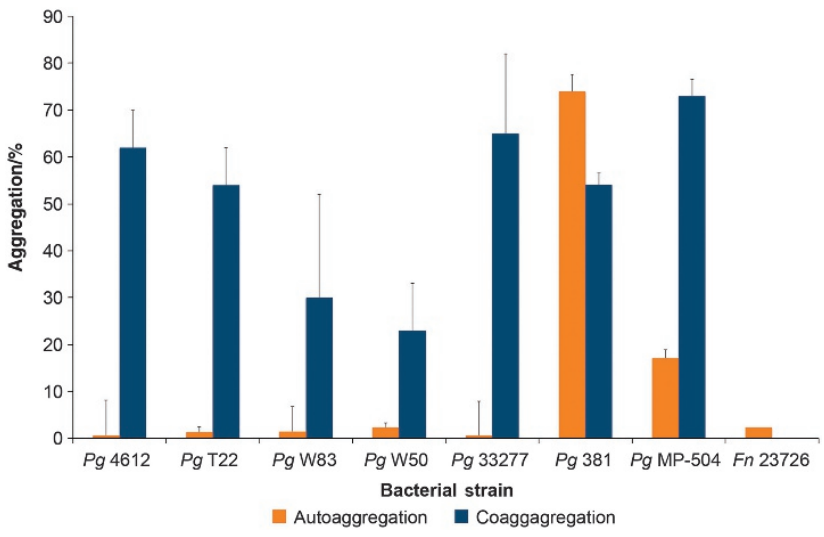

Figure 1 Quantitative autoaggregation levels of bacterial strains and coaggregation levels between Fusobacterium nucleatum ATCC 23726 and seven different Porphyromonas gingivalis strains. Data are expressed as percentage aggregation and represent the means and standard deviation of at least three independent experiments.

different $P$. gingivalis strains tested in this study exhibited a diverse inhibition profile that involved strain-dependent sensitivities to the inhibitors tested (Figure 2). Attachment to P. gingivalis strains 4612 and T22 was partially sensitive to arginine, galactose and lactose, whereas coaggregation with ATCC 33277 was almost completely abolished by the addition of arginine alone and partially sensitive to lactose. Coaggregation reactions in the presence of $50 \mathrm{mmol} \cdot \mathrm{L}^{-1}$ arginine resulted in a relative decrease in coaggregation by $62 \% \pm$ $2 \%$ with $4612,70 \% \pm 6 \%$ with T22 and $89 \% \pm 3 \%$ with ATCC 33277 compared with the corresponding reactions with ATCC 23726 in the absence of inhibitor. Reactions between F. nucleatum ATCC 23726 and $P$. gingivalis strains in the presence of galactose revealed, a $58 \% \pm$ $16 \%$ decrease in coaggregation with 4612 and $36 \% \pm 18 \%$ reduction of binding with T22 compared with control reactions without inhibitor. Coaggregation in the presence of $50 \mathrm{mmol} \cdot \mathrm{L}^{-1}$ lactose resulted in a relative decrease in coaggregation by $46 \% \pm 13 \%$ with $4612,56 \% \pm 7 \%$ with T22 and $40 \% \pm 8 \%$ with ATCC 33277 compared with the corresponding reactions with ATCC 23726 in the absence of inhibitor. Interactions between ATCC 23726 and ATCC 33277 were not affected by galactose, with coaggregation being reduced by only $12 \% \pm 6 \%$ compared with the strains reacting in $\mathrm{CAB}$. Addition of glucose or glutamic acid did not affect adhesion of any of the strain combinations tested.

\section{Fusobacterial OMPs function as adhesins for interaction with $P$. gingivalis}

In a previous study, we created gene inactivation mutants in large outer membrane autotransporter proteins (OMPs) of F. nucleatum ATCC 23726 and identified one of them, RadD, as an adhesin for interaction with Gram-positive Streptococci and Actinomyces. ${ }^{13}$ Recently, an additional, one of these large OMPs, Fap2 was characterized as the adhesin for galactose-inhibitable binding of $P$. gingivalis $\mathrm{PK}$ 1924 to the same strain, ATCC 23726, of F. nucleatum used here. ${ }^{14}$ As we observed strain-dependent coaggregation profiles in our inhibition experiments, we examined the binding of the previously generated OMP mutant panel, including the arginine-inhibitable RadD and galactose-inhibitable Fap2 to the three different $P$. gingivalis strains (4612, T22 and ATCC 33277) that exhibited significant binding in this study to the parent strain ATCC 23726 (Figure 3). Similar to the inhibition results, coaggregation between the F. nucleatum ATCC 23726 OMP mutant derivatives and the $P$. gingivalis strains varied 

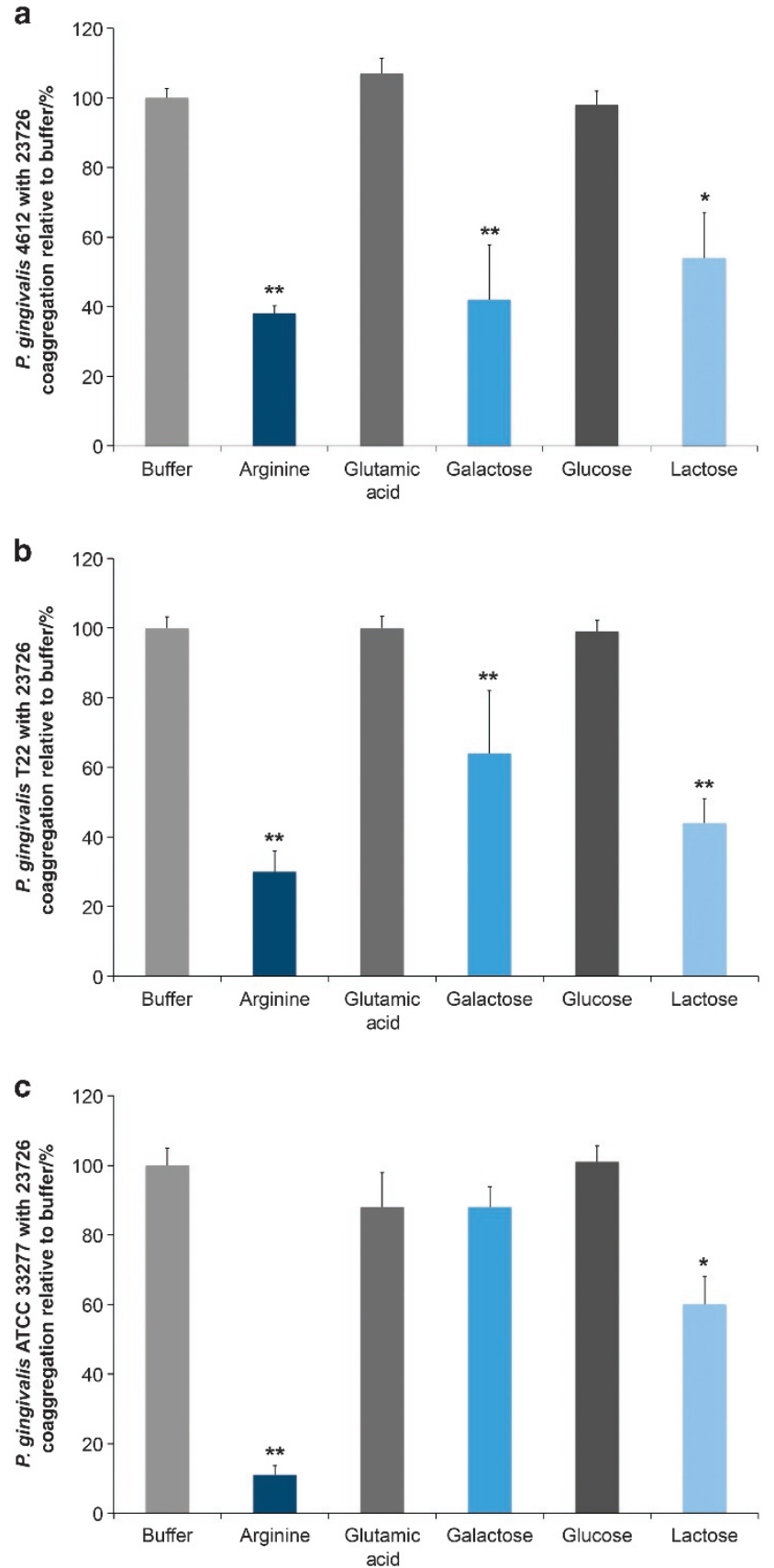

Figure 2 Quantitative inhibition of coaggregation assay between Fusobacterium nucleatum ATCC 23726 and Porphyromonas gingivalis strains in the presence of inhibitors. (a) $P$. gingivalis 4612 with $F$. nucleatum ATCC 23726; (b) $P$. gingivalis T22 with $F$. nucleatum ATCC 23726; (c) $P$. gingivalis ATCC 33277 with $F$. nucleatum ATCC 23726. Data are expressed as relative percentage coaggregation compared to coaggregation reaction of the partner strains in buffer set as $100 \%$ and represent the means and standard deviation of at least three independent experiments. ${ }^{*} P<0.05 ;{ }^{*} P<0.01$.

depending on the interacting pairs. Fusobacterial interaction with $P$. gingivalis strain 4612 appears to be mediated by both Fap2 and RadD, with the average relative decrease for the individual mutants (Fap2 mutant, $53 \% \pm 6 \%$ and RadD mutant, $29 \% \pm 6 \%$ ) adding up to the decrease seen for the Fap $2 /$ RadD double mutant $(82 \% \pm 5 \%)$. Among the OMP mutants tested here, only lack of Fap2 resulted in a reduction of the interaction between F. nucleatum ATCC 23726 and
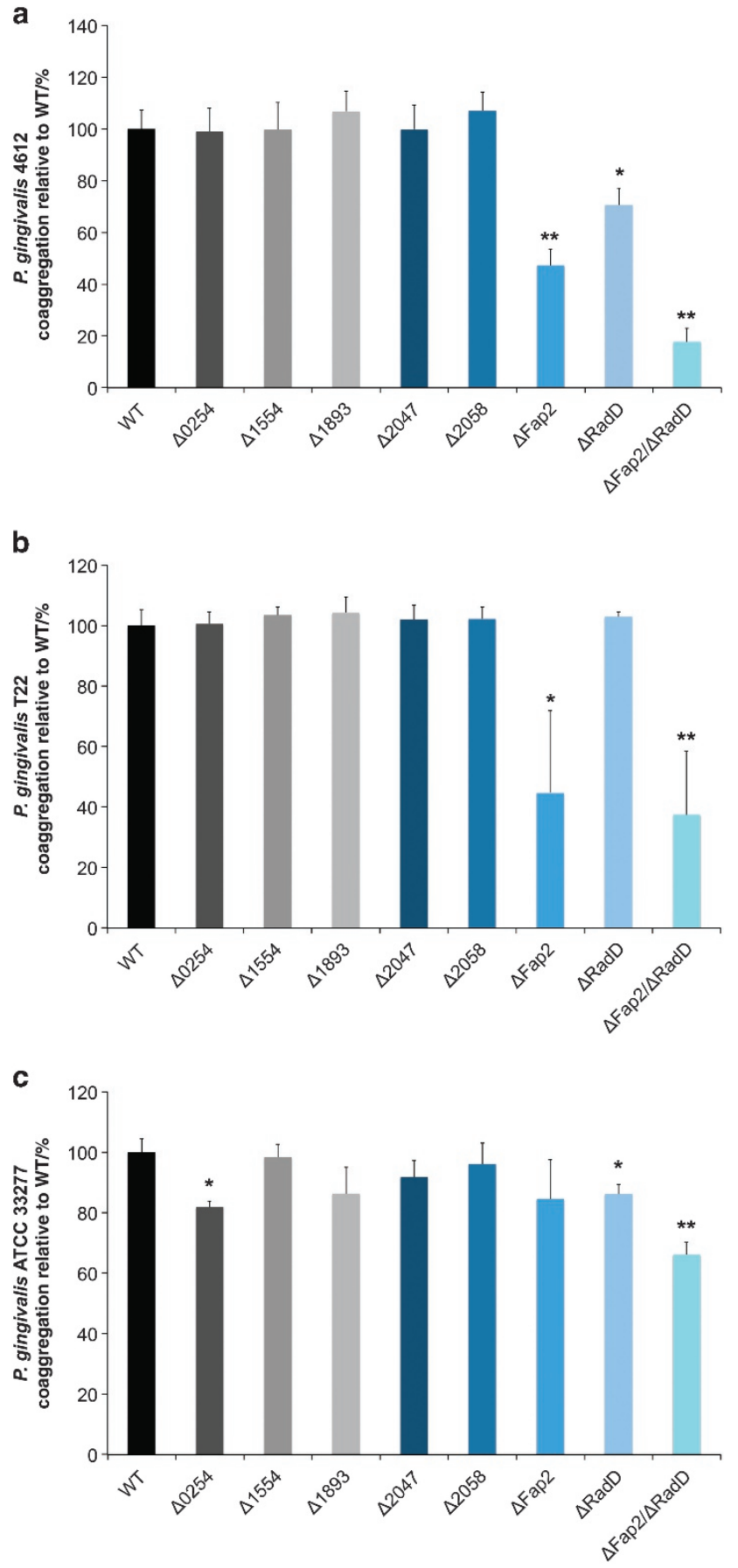

Figure 3 Quantitative coaggregation assay between Fusobacterium nucleatum ATCC 23726 and mutant derivatives in outer membrane proteins with Porphyromonas gingivalis strains. (a) P. gingivalis 4612 with $F$. nucleatum ATCC 23726; (b) P. gingivalis T22 with $F$. nucleatum ATCC 23726; (c) P. gingivalis ATCC 33277 with F. nucleatum ATCC 23726. Data are expressed as relative percentage coaggregation compared with coaggregation reaction of the wild type with the respective $P$. gingivalis partner strains set as $100 \%$. Data represent the means and standard deviation of at least three independent experiments. ${ }^{*} P<0.05 ;{ }^{*} P<0.01$. WT, wild type.

$P$. gingivalis T22 with a decrease of $55 \% \pm 27 \%$ relative to coaggregation with the wild-type parent strain. Binding to P. gingivalis 33277 displayed only a slight decrease with several of the OMP mutants tested. However, with the exception of the decrease $(34 \% \pm 4 \%)$ 


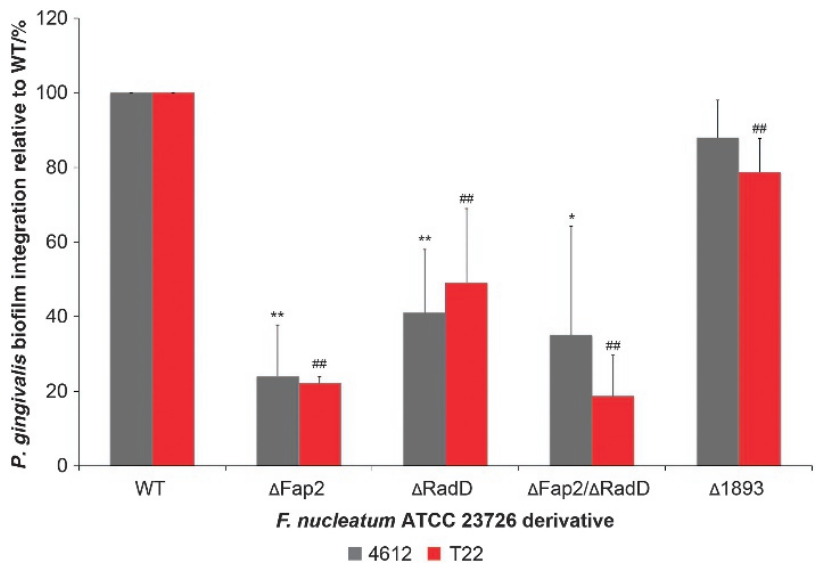

Figure 4 Porphyromonas gingivalis integration in dual-species biofilms with Fusobacterium nucleatum ATCC $23726 \Delta$ Fap2, $\Delta$ RadD and $\Delta$ Fap2/ $\Delta$ RadD outer membrane proteins mutant derivatives. Biofilm integration is given as a percentage relative to biofilm integration measured with WT $F$. nucleatum ATCC 23726. At least three independent experiments were performed per strain combination. Data represent the means and standard deviation of at least three independent experiments. ${ }^{*} P<0.05$; ${ }^{*} P<0.01$; $\# \#<<0.01$. WT, wild type.

observed with the Fap2/RadD double mutant, none of the apparent reductions were significant.

\section{Biofilm integration}

Previous studies have shown that the same strains of bacteria grown under biofilms conditions reveal different gene expression and transcriptomic patterns when compared with their planktonically grown counterparts. ${ }^{43-45}$ As our coaggregation experiments are typically performed with planktonically grown cells, we wanted to confirm that the differences seen in coaggregation patterns with the F. nucleatum OMP mutants is relevant for the integration of $P$. gingivalis strains 4612 and T22 into pre-existing fusobacterial biofilms. P. gingivalis strain 33277 was not included in the biofilm studies because coaggregation reactions with $F$. nucleatum OMP mutants did not show significant involvement of these adhesins in this interaction.

Our dual-species biofilm studies were conducted with $P$. gingivalis 4612 and T22 and revealed significant reduction in $P$. gingivalis 4612 integration into a biofilm when grown with $F$. nucleatum derivatives lacking Fap2 (24\% $\pm 14 \%$ integration), RadD (41\% $\pm 17 \%$ integration), or the Fap $2 /$ RadD double mutant $(35 \% \pm 29 \%$ integration $)$ when compared with biofilms containing wild-type F. nucleatum. $P$. gingivalis T22 exhibited a similar pattern of biofilm integration as 4612 with significantly decreased integration when grown with Fap2 $(22 \% \pm 2 \%$ integration), $\operatorname{RadD}(49 \% \pm 20 \%$ integration $)$ or the Fap2/RadD double mutant $(19 \% \pm 11 \%$ integration; Figure 4$)$. Biofilm growth with the F. nucleatum ATCC 23726 mutant derivative carrying a deletion in OMP FN1893 served as a control to ensure that decreased $P$. gingivalis integration in the dual-species biofilms were mutation specific and not the result of a general biofilm phenotype effect caused by lack of an OMP.

\section{DISCUSSION}

In this study, we provide evidence that in addition to the previously described Fap2-mediated interaction between F. nucleatum ATCC 23726 and $P$. gingivalis strain PK $1924,{ }^{14}$ multiple adhesins have a role in the attachment of this fusobacterial strain with $P$. gingivalis in a highly strain-dependent manner. The notion of isolate-specific interaction is not new and has been observed for a number of different species pairs including F. nucleatum with a variety of Selemonas, Streptococcus and Actinomyces species as well as different isolates of $P$. gingivalis and other oral bacteria. ${ }^{2,26,46}$ Other studies describe similar phenomena of strain-specific interactions for Actinomyces species with different Streptococci $i^{25,28}$ as well as a number of additional oral bacterial species. ${ }^{29-30,47}$ In addition to Fap2, several adhesins involved in some of these interactions have been identified including SspA/B in the binding of $S$. gordonii with $P$. gingivalis $^{48}$ and the fusobacterial RadD in the interaction between $F$. nucleatum and Streptococcal species as well as Actinomyces. ${ }^{13}$ However, most studies have only investigated individual strains for each of the species involved and comprehensive studies including multiple isolates are still lacking. Considering the previously observed strain-dependent variations as well as the observation that the inhibition profiles of interactions between species can depend on isolates or serotypes tested, the presence of additional adhesins would be expected.

Quantitative coaggregation studies between F. nucleatum ATCC 23726 and different strains of $P$. gingivalis revealed robust coaggregation with strains, 4612, T22 and ATCC 33277, but a relatively weak interaction with strains W50 and W83 (Figure 1). This differential affinity between $F$. nucleatum and the periodontal pathogen $P$. gingivalis could have a role in virulence of oral biofilms. W50 and W83 are highly pathogenic strains of $P$. gingivalis, ${ }^{49-50}$ and the type of $F$. nucleatum strain already present in the oral biofilm could determine whether more or less virulent variants of $P$. gingivalis integrate into the biofilm. For example, W83 has been shown to associate with $F$. nucleatum in oral epithelial cell invasion ${ }^{51}$ and F. nucleatum clinical isolate TDC100 enhanced invasion significantly more than F. nucleatum ATCC 25586. Thus, understanding which adhesins are involved in interactions with more virulent pathogens could shed light on the molecular mechanisms of pathogenic biofilm formation.

Similar to previous observations for binding of F. nucleatum to Streptococci, ${ }^{52}$ the interactions between F. nucleatum ATCC 23726 and at least two of the three strongly binding strains of $P$. gingivalis is multimodal, as substantial levels of coaggregation remained even in the presence of inhibitors (Figure 2). Consistent with a strain-dependent multi-modal interaction, binding of $F$. nucleatum to $P$. gingivalis was mediated through adhesins sensitive to arginine, galactose and lactose at varying degrees. This was especially interesting because historically, fusobacterial adherence to the predominantly Gramnegative late oral colonizers, including $P$. gingivalis, has largely been associated with galactose-inhibitable interactions, while coaggregation with Gram-positive early colonizing species are suggested to be mediated by arginine-inhibitable interactions. ${ }^{26}$ In contrast to the binding characterized between F. nucleatum ATCC 23726 and P. gingivalis strain PK 1924, ${ }^{14}$ which followed this paradigm, the attachment of the same fusobacterial strain to the $P$. gingivalis derivatives tested in this study was only partially sensitive to the addition of galactose (4612 and T22) or not at all (ATCC 33277). Surprisingly, arginine had a stronger effect on the coaggregation with all three $P$. gingivalis strains with the binding to ATCC 33277 being almost completely abolished by the addition of this amino acid (Figure 2c). Lactose-inhibition was observable in coaggregation with all three $P$. gingivalis strains used in this study and were consistent with previous findings that suggest lactose-inhibitable coaggregations may be a common form of interaction among changing populations of bacteria in the shift from health to a state of severe periodontal disease. ${ }^{2,26}$ 
Screening of the autotransporter large OMP mutant collection from a previous study, ${ }^{13}$ revealed that in addition to Fap2, RadD functions as one of the adhesins mediating the binding between $F$. nucleatum ATCC 23726 and $P$. gingivalis 4612 (Figure 3a). This was unexpected because RadD was previously identified as a major arginine-inhibitable adhesin for interactions of $F$. nucleatum with several Gram-positive species. ${ }^{12-13}$ Among the OMP mutant collection, only lack of Fap2 resulted in a partial reduction $(\sim 50 \%)$ of coaggregation with $P$. gingivalis strain T22 indicating that the additional adhesin that contributes to the interaction between these strains constitutes a different type of cell surface feature. This unidentified adhesin is likely to provide the arginine-inhibitable feature of the interaction, as Fap2mediated adhesion to $P$. gingivalis has been described previously as galactose-inhibitable. ${ }^{14}$ Both RadD and Fap2 are multifunctional OMPs that were previously characterized for their role in induction of cell death in human lymphocytes. ${ }^{24}$ RadD has additional functions as the above-mentioned arginine-inhibitable adhesin for attachment to Gram-positive early colonizers ${ }^{12-13}$ and the newly discovered role as one of the adhesins for attachment to $P$. gingivalis strain T22. Multifunctionality has been described for other large OMPs including TolC of Escherichia coli, ${ }^{53}$ OprF for Pseudomonas ${ }^{54}$ and the major OMP of Campylobacter jejuni. ${ }^{55}$ The arginine-inhibitable adhesin that largely mediates the coaggregation of F. nucleatum ATCC 23726 with $P$. gingivalis ATCC 33277, which could also contribute the argininesensitive attachment to T22 has yet to be identified in F. nucleatum.

Our results for integration of $P$. gingivalis 4612 and T22 into fusobacterial biofilms formed by F. nucleatum ATCC 23726 wild-type and its mutant derivative lacking Fap2, RadD or both as well as FN1893 confirmed the importance of Fap2 and RadD for attachment of 4612 to ATCC 23726 (Figure 4). Under biofilm growth conditions RadD may also have a role in binding between F. nucleatum ATCC 23726 and $P$. gingivalis T22. This additional function of RadD was not apparent in the interaction with T22 and could be due to differential expression of adhesins in this strain of $P$. gingivalis under biofilm growth conditions. Adhesins are critical virulence factors whose expression are regulated and coordinated to ensure that the necessary adhesin is expressed at the right time. ${ }^{56-57}$ When bacterial species have integrated into existing biofilms, significant changes come about compared with their planktonic counterparts including gene expression patterns and physiological properties. ${ }^{45,58}$

In summary, we report that interspecies interactions between $F$. nucleatum and $P$. gingivalis involve a number of different fusobacterial adhesins. The previously characterized Fap2 (Coppenhagen-Glazer, 2015) appears to be a more prominent adhesin that takes part in the attachment to three of the four $P$. gingivalis strains investigated so far for their attachment to F. nucleatum on a molecular level. The arginine-inibitable adhesin RadD, which we originally identified as a major adhesin for interaction with streptococci and other early colonizers $^{12-13}$ contributes to the attachment to at least one strain of $P$. gingivalis and possibly a second one under biofilm growth conditions. In addition, we provide evidence for the presence of at least one additional adhesin that is sensitive to arginine and is not a member of the autotransporter family type of fusobacterial large OMPs. These findings are consistent with earlier observations of different coaggregation groups for $F$. nucleatum with $P$. gingivalis that vary in their sensitivity to a variety of inhibitors. ${ }^{26,59}$ Because certain strain-strain interactions could be more pathogenic than others, we believe that an improved understanding of the array of adhesins involved in interspecies attachment will continue to clarify the role of F. nucleatum in health and disease. ${ }^{39}$

\section{ACKNOWLEDGEMENTS}

Susan K Haake passed away before the submission of the final version of this manuscript. Renate Lux accepts responsibility for the integrity and validity of the data collected and analyzed. Renate Lux was supported by National Institute of Health, National Institute of Dental and Craniofacial Research DE021108 and DE018276. Jane Park was supported by NIDCR grant 5T90DE022734-02.

1 Andersen RN, Ganeshkumar N, Kolenbrander PE. Helicobacter pylori adheres selectively to Fusobacterium spp. Oral Microbiol Immunol 1998; 13(1): 51-54.

2 Kolenbrander PE, Andersen RN, Moore LV. Coaggregation of Fusobacterium nucleatum, Selenomonas flueggei, Selenomonas infelix, Selenomonas noxia, and Selenomonas sputigena with strains from 11 genera of oral bacteria. Infect Immun 1989; 57(10): 3194-3203

3 Kolenbrander PE, Parrish KD, Andersen RN et al. Intergeneric coaggregation of oral Treponema spp. with Fusobacterium spp. and intrageneric coaggregation among Fusobacterium spp. Infect Immun 1995; 63(12): 4584-4588.

4 Berezow AB, Darveau RP. Microbial shift and periodontitis. Periodontol 2000 2011; 55(1): 36-47.

5 Han YW, Wang X. Mobile microbiome: oral bacteria in extra-oral infections and inflammation. J Dent Res 2013; 92(6): 485-491.

6 Han YW. Fusobacterium nucleatum: a commensal-turned pathogen. Curr Opin Microbiol 2015; 23: 141-147.

7 Swidsinski A, Dörffel Y, Loening-Baucke V et al. Acute appendicitis is characterised by local invasion with Fusobacterium nucleatum/necrophorum. Gut 2011; 60(1): 3440 .

8 Témoin S, Chakaki A, Askari A et al. Identification of oral bacterial DNA in synovial fluid of patients with arthritis with native and failed prosthetic joints. J Clin Rheumato/ 2012; 18(3): 117-121.

9 Chaim W, Mazor M. Intraamniotic infection with fusobacteria. Arch Gynecol Obstet 1992; 251(1): 1-7.

10 Han YW, Fardini Y, Chen C et al. Term stillbirth caused by oral Fusobacterium nucleatum. Obstet Gynecol 2010; 115(2 Pt 2): 442-445.

11 Bradshaw DJ, Marsh PD, Watson GK et al. Role of Fusobacterium nucleatum and coaggregation in anaerobe survival in planktonic and biofilm oral microbial communities during aeration. Infect Immun 1998; 66(10): 4729-4732.

12 Kaplan A, Kaplan CW, He X et al. Characterization of aid1, a novel gene involved in Fusobacterium nucleatum interspecies interactions. Microb Ecol 2014; 68(2): 379387.

13 Kaplan CW, Lux R, Haake SK et al. The Fusobacterium nucleatum outer membrane protein RadD is an arginine-inhibitable adhesin required for inter-species adherence and the structured architecture of multispecies biofilm. Mol Microbiol 2009; 71(1): 35-47.

14 Coppenhagen-Glazer S, Sol A, Abed J et al. Fap2 of Fusobacterium nucleatum is a galactose-inhibitable adhesin involved in coaggregation, cell adhesion, and preterm birth. Infect Immun 2015; 83(3): 1104-1113.

15 Henderson IR, Navarro-Garcia F, Desvaux M et al. Type $V$ protein secretion pathway: the autotransporter story. Microbiol Mol Biol Rev 2004; 68(4): 692-744.

16 Bullard B, Lipski S, Lafontaine ER. Regions important for the adhesin activity of Moraxella catarrhalis Hag. BMC Microbiol 2007; 7: 65.

17 Lipski SL, Akimana C, Timpe JM et al. The Moraxella catarrhalis autotransporter McaP is a conserved surface protein that mediates adherence to human epithelial cells through its N-terminal passenger domain. Infect Immun 2007; 75(1): 314 324.

18 Heras B, Totsika M, Peters KM et al. The antigen 43 structure reveals a molecular Velcro-like mechanism of autotransporter-mediated bacterial clumping. Proc Natl Acad Sci USA 2014; 111(1): 457-462.

19 Klemm P, Hjerrild L, Gjermansen M et al. Structure-function analysis of the selfrecognizing antigen 43 autotransporter protein from Escherichia coli. Mol Microbiol 2004; 51(1): 283-296.

20 Sherlock 0, Schembri MA, Reisner A et al. Novel roles for the AIDA adhesin from diarrheagenic Escherichia coli: cell aggregation and biofilm formation. J Bacteriol 2004; 186(23): 8058-8065.

21 Valle J, Mabbett AN, Ulett GC et al. UpaG, a new member of the trimeric autotransporter family of adhesins in uropathogenic Escherichia coli. J Bacterio/ 2008; 190 (12): 4147-4161.

22 Capecchi B, Adu-Bobie J, Di Marcello F et al. Neisseria meningitidis NadA is a new invasin which promotes bacterial adhesion to and penetration into human epithelial cells. Mol Microbiol 2005; 55(3): 687-698.

23 Kaplan CW, Lux R, Huynh T et al. Fusobacterium nucleatum apoptosis-inducing outer membrane protein. J Dent Res 2005; 84(8): 700-704.

24 Kaplan CW, MaX, Paranjpe A et al. Fusobacterium nucleatum outer membrane proteins Fap2 and RadD induce cell death in human lymphocytes. Infect Immun 2010; 78(11): 4773-4778.

25 Cisar JO, Kolenbrander PE, McIntire FC. Specificity of coaggregation reactions between human oral streptococci and strains of Actinomyces viscosus or Actinomyces naes/undii. Infect Immun 1979; 24(3): 742-752. 
26 Kolenbrander PE, Andersen RN. Inhibition of coaggregation between Fusobacterium nucleatum and Porphyromonas (Bacteroides) gingivalis by lactose and related sugars. Infect Immun 1989; 57(10): 3204-3209.

27 Crowley PJ, Fischlschweiger W, Coleman SE et al. Intergeneric bacterial coaggregations involving mutans streptococci and oral actinomyces. Infect Immun 1987; 55(11): 2695-2700.

28 Jakubovics NS, Strömberg N, van Dolleweerd CJ et al. Differential binding specificities of oral streptococcal antigen I/II family adhesins for human or bacterial ligands. Mol Microbiol 2005; 55(5): 1591-1605.

29 Kolenbrander PE, Inouye Y, Holdeman LV. New Actinomyces and Streptococcus coaggregation groups among human oral isolates from the same site. Infect Immun 1983; 41(2): 501-506.

30 Eke PI, Rotimi VO, Laughon BE. Coaggregation of black-pigmented Bacteroides species with other oral bacteria. J Med Microbiol 1989; 28(1): 1-4.

31 Kolenbrander PE, Andersen RN, Moore LV. Intrageneric coaggregation among strains of human oral bacteria: potential role in primary colonization of the tooth surface. Appl Environ Microbiol 1990; 56(12): 3890-3894.

32 Noguchi N, Noiri Y, Narimatsu M et al. Identification and localization of extraradicular biofilm-forming bacteria associated with refractory endodontic pathogens. Appl Environ Microbiol 2005; 71(12): 8738-8743.

33 Ximénez-Fyvie LA, Haffajee AD, Socransky SS. Microbial composition of supra- and subgingival plaque in subjects with adult periodontitis. J Clin Periodontol 2000; 27(10): 722-732.

34 Lamont RJ, Hersey SG, Rosan B. Characterization of the adherence of Porphyromonas gingivalis to oral streptococci. Oral Microbiol Immunol 1992; 7: 193-197.

35 Kinder SA, Holt SC. Characterization of coaggregation between Bacteroides gingivalis T22 and Fusobacterium nucleatum T18. Infect Immun 1989; 57(11): 3425-3433.

36 Lamont RJ, Chan A, Belton CM et al. Porphyromonas gingivalis invasion of gingival epithelial cells. Infect Immun 1995; 63(10): 3878-3885.

37 Ansai T, Yamashita Y, Awano S et al. A murC gene in Porphyromonas gingivalis 381. Microbiology 1995; 141(Pt 9): 2047-2052.

38 Shah HN, Williams RA, Bowden GH et al. Comparison of the biochemical properties of Bacteroides melaninogenicus from human dental plaque and other sites. J Appl Bacteriol 1976; 41(3): 473-495.

39 Kinder SA, Holt SC. Coaggregation between bacterial species. Meth Enzymol 1994; 236: $254-270$.

40 Saito Y, Fujii R, Nakagawa KI et al. Stimulation of Fusobacterium nucleatum biofilm formation by Porphyromonas gingivalis. Oral Microbiol Immunol 2008; 23(1): $1-6$.

41 Takemoto T, Ozaki M, Shirakawa M et al. Purification of arginine-sensitive hemagglutinin from Fusobacterium nucleatum and its role in coaggregation. J Periodont Res 1993; 28(1): 21-26.

42 Shaniztki B, Hurwitz D, Smorodinsky N et al. Identification of a Fusobacterium nucleatum PK1594 galactose-binding adhesin which mediates coaggregation with periopathogenic bacteria and hemagglutination. Infect Immun 1997; 65(12): 5231-5237.

43 Becker P, Hufnagle W, Peters G et al. Detection of differential gene expression in biofilm-forming versus planktonic populations of Staphylococcus aureus using micro-representational-difference analysis. Appl Environ Microbiol 2001; 67(7): 29582965.

44 Lo A, Seers C, Dashper S et al. FimR and Fims: biofilm formation and gene expression in Porphyromonas gingivalis. J Bacteriol 2010; 192(5): 1332-1343.
45 Resch A, Rosenstein R, Nerz C et al. Differential gene expression profiling of Staphylococcus aureus cultivated under biofilm and planktonic conditions. Appl Environ Microbiol 2005; 71(5): 2663-2676.

46 Biyikoğlu B, Ricker A, Diaz PI. Strain-specific colonization patterns and serum modulation of multi-species oral biofilm development. Anaerobe 2012; 18(4): 459-470.

47 Kolenbrander PE, Williams BL. Prevalence of viridans streptococci exhibiting lactoseinhibitable coaggregation with oral actinomycetes. Infect Immun 1983; 41(2): 449452.

48 Lamont RJ, El-Sabaeny A, Park Y et al. Role of the Streptococcus gordonii SspB protein in the development of Porphyromonas gingivalis biofilms on streptococcal substrates. Microbiology 2002; 148(Pt 6): 1627-1636.

49 Grenier D, Mayrand D. Selected characteristics of pathogenic and nonpathogenic strains of Bacteroides gingivalis. J Clin Microbiol 1987; 25(4): 738-740.

50 Neiders ME, Chen PB, Suido $\mathrm{H}$ et al. Heterogeneity of virulence among strains of Bacteroides gingivalis. J Periodont Res 1989; 24(3): 192-198.

51 Saito A, Inagaki S, Kimizuka R et al. Fusobacterium nucleatum enhances invasion of human gingival epithelial and aortic endothelial cells by Porphyromonas gingivalis. FEMS Immunol Med Microbiol 2008; 54(3): 349-355.

52 Takemoto T, Hino T, Yoshida M et al. Characteristics of multimodal co-aggregation between Fusobacterium nucleatum and streptococci. J Periodont Res 1995; 30(4): 252-257.

53 Werner J, Augustus AM, Misra R. Assembly of TolC, a structurally unique and multifunctional outer membrane protein of Escherichia coli K-12. J Bacteriol 2003; 185(22): 6540-6547.

54 Bodilis J, Barray S. Molecular evolution of the major outer-membrane protein gene (oprF) of Pseudomonas. Microbiology 2006; 152(Pt 4): 1075-1088.

55 Schröder W, Moser I. Primary structure analysis and adhesion studies on the major outer membrane protein of Campylobacter jejuni. FEMS Microbiol Lett 1997; 150(1): $141-147$.

56 Engstrom MD, Alteri CJ, Mobley HL. A conserved PapB family member, TosR, regulates expression of the uropathogenic Escherichia coli RTX nonfimbrial adhesin TosA while conserved LuxR family members TosE and TosF suppress motility. Infect Immun 2014; 82(9): 3644-3656.

57 Sievers S, Sternkopf Lillebæk EM, Jacobsen K et al. A multicopy sRNA of Listeria monocytogenes regulates expression of the virulence adhesin LapB. Nucleic Acids Res 2014; 42(14): 9383-9398.

58 Cook GS, Costerton JW, Lamont RJ. Biofilm formation by Porphyromonas gingivalis and Streptococcus gordonii. J Periodont Res 1998; 33(6): 323-327.

59 Metzger Z, Blasbalg J, Dotan M et al. Characterization of coaggregation of Fusobacterium nucleatum PK1594 with six Porphyromonas gingivalis strains. J Endod 2009; 35(1): 50-54.

(c) (i) $\ominus$ This work is licensed under a Creative Commons Attributionc. $\mathrm{BY}$ NC ND NonCommercial-NoDerivs 4.0 International License. The images or other third party material in this article are included in the article's Creative Commons license, unless indicated otherwise in the credit line; if the material is not included under the Creative Commons license, users will need to obtain permission from the license holder to reproduce the material. To view a copy of this license, visit http:// creativecommons.org/licenses/by-nc-nd/4.0/

(C) The Author(s) 2016

Supplementary Information for this article can be found on the International Journal of Oral Science website (http://www.nature.com/ijos) 